\title{
Comportamiento de los trastornos temporomandibulares en pacientes mayores de 60 años. Amancio, 2018.
}

\author{
Behavior of temporomandibular disorders in patients \\ over 60 years old. Amancio, 2018.
}

\author{
Licet Abreu-García,* Carmen García-Alguacil ${ }^{\ddagger}$
}

\section{RESUMEN}

El artículo resume los resultados de una investigación observacional descriptiva de corte transversal, realizada en el consultorio No. 19 perteneciente al área de salud de la Clínica Estomatológica del Hospital Docente «Dr. Luis Aldana Palomino» Municipio Amancio Rodríguez, Las Tunas, Cuba, en el periodo comprendido entre octubre de 2017 y junio de 2019, cuyo objetivo fue describir el comportamiento de los trastornos temporomandibulares en pacientes mayores de 60 años, con un universo de 120 pacientes diagnosticados y la muestra aleatoria de 90 de ellos. En los resultados se destacó que el rango de edad entre 60 y 65 años y en sexo femenino fue donde más prevalecieron los trastornos temporomandibulares; el que se presentó con más frecuencia fue el muscular grupo I, según la clasificación de Okeson. De los factores etiológicos oclusales encontrados, el bruxismo y la rehabilitación protésica fueron los más representativos. El desgaste dentario fue el parámetro clínico más reportado de forma general presentándose en ambos tipos de trastornos.

Palabras clave: Trastornos temporomandibulares, adulto mayor.

\section{ABSTRACT}

The article sums up the results of an observational, descriptive, and transversal study, carried out at the dispensary 19, belonging to the area of health of the Stomatological Clinic in the educational hospital «Dr. Luis Aldana Palomino» in the municipality Amancio Rodríguez, Las Tunas, Cuba, in the period between October 2017 and June 2019, whose objective was to describe the development of temporomandibular disorders in $60+$ year-old patients, from a universe of 120 patients diagnosed and a random sample of 90 of them. In the results, it is highlighted that the age range between 60 and 65 years and female patients was where most of the temporomandibular disorders occurred, being the most frequent the muscular group I, per the Okeson classification. Of the occlusal etiologic factors found, bruxism and prosthetic rehabilitation were the most representative. Dental wear was the clinical parameter most reported in general, showing up in both types of disorders.

Keywords: Temporomandibular disorders, older adult.

\section{INTRODUCCIÓN}

$\mathrm{E}^{\prime}$ | envejecimiento es un proceso natural de duración Evariable, homogéneo para cada especie, en el que influyen numerosos factores condicionantes, algunos propios del individuo como los genéticos y otros externos como los ambientales o circunstanciales. ${ }^{1}$
Los adultos mayores requieren atención priorizada y especializada de toda la sociedad, por lo que se debe tener un conocimiento más sólido en cuanto a los aspectos biológicos, psicológicos y sociales relacionados con la vejez. ${ }^{2}$

El abordaje de los trastornos temporomandibulares (TTM) en los adultos mayores se ha diferenciado muy poco respecto a otros grupos de edades. ${ }^{3}$

\footnotetext{
* Residente de segundo año. Especialidad Estomatología General Integral.

¥ Especialista en primer grado prótesis. Profesor auxiliar. Clínica Estomatológica.

Hospital Clínico Quirúrgico «Dr. Luis Aldama Palomino».

Recibido: 21 mayo 2019. Aceptado para publicación: 07 septiembre 2020.
}

Citar como: Abreu-García L, García-Alguacil C. Comportamiento de los trastornos temporomandibulares en pacientes mayores de 60 años. Amancio, 2018. Revista ADM 2020; 77 (5): 239-243. https://dx.doi.org/10.35366/96141 
La mayoría de las investigaciones se encamina al diagnóstico clínico y epidemiológico, ya sea en adultos mayores institucionalizados o como parte de la población general; y dejan de lado las posibles alternativas de una atención integral que abarque y controle todos los elementos que constituyen riesgos de desencadenar, empeorar o perpetuar los TTM en adultos mayores. ${ }^{4}$

En Cuba demográficamente existe una pirámide poblacional que muestra una elevada tendencia a aplanarse en su cúspide a consecuencia de una marcada disminución en la fecundidad, un incremento significativo de la esperanza de vida al nacer y un incremento de las migraciones a expensas de poblaciones jóvenes, lo cual hace más representativo este grupo poblacional. ${ }^{5}$

Existen varias investigaciones que tratan sobre los TTM en nuestro país, pero sobre todo la prevalencia de éstos en jóvenes, dentados, pero no se conocen amplias referencias de este tipo de estudio en el adulto mayor. ${ }^{6}$

En la provincia de Las Tunas ha ido aumentando la tasa en el comportamiento de los trastornos temporomandibulares en pacientes mayores de 60 años, lo que ha motivado la realización de esta investigación. El desconocimiento de las personas, la falta de asistencia temprana y oportuna a las consultas de estomatología como también la falta de estudios dirigidos han influido en esta problemática.

Son los resultados de las investigaciones en epidemiología gerontológica los que posibilitan la formulación, evaluación, y aplicación de políticas y programas de salud, los que ofrecen una base científica sólida que permite mejorar el bienestar y la calidad de vida, los que aportan todo el conocimiento relacionado con el envejecimiento y hacen posible un uso más eficaz de los recursos destinados a los adultos mayores.

\section{MATERIAL Y MÉTODOS}

Se realizó un estudio observacional, descriptivo, de corte transversal en pacientes mayores de 60 años del Consultorio No. 19 del área perteneciente a la Clínica de Estomatología del Hospital Docente "Luis Aldana Palomino", Municipio Amancio Rodríguez, Las Tunas, con el objetivo de describir el comportamiento de los trastornos temporomandibulares en el periodo comprendido entre octubre de 2017 y junio de 2019.

\section{Universo y muestra de estudio}

El universo de estudio lo conformó un total de 120 personas mayores de 60 años con trastornos temporomandibulares diagnosticados y para la muestra seleccionada se llevó a cabo un muestreo aleatorio simple de 90 de ellos, los cuales cumplían los siguientes:

Criterios de inclusión:

- Pacientes mayores de 60 años de edad dispuestos a participar en el estudio previo consentimiento informado.

- Pacientes que no presentaban discapacidad mental o cognitiva que les imposibilitara y/o comprometiera la calidad de la realización de la entrevista.

Criterios de exclusión:

- Pacientes que en el periodo en que se realizó la investigación estuvieron fuera del municipio.

- Pacientes politraumatizados que presentaron trastornos articulares agudos como secuelas del trauma.

El interrogatorio y el examen físico fueron realizados por la residente de la especialidad de estomatología general in-

Tabla 1: Trastornos temporomandibulares.

\begin{tabular}{lcccc}
$\begin{array}{l}\text { Edad } \\
\text { (años) }\end{array}$ & $\begin{array}{c}\text { Trastorno muscular (I) } \\
\mathrm{n}(\%)\end{array}$ & $\begin{array}{c}\text { Trastorno articular (II) } \\
\mathrm{n}(\%)\end{array}$ & $\begin{array}{c}\text { Trastorno hipomovilidad (III) } \\
\mathrm{n}(\%)\end{array}$ & $\begin{array}{c}\text { Total } \\
\mathrm{n}(\%)\end{array}$ \\
\hline 60 a 65 & $26(28.9)$ & $10(11.1)$ & $1(1.1)$ & $37(41.2)$ \\
66 a 70 & $29(32.2)$ & $4(4.4)$ & $0(0.0)$ & $33(36.7)$ \\
71 a 75 & $9(10.0)$ & $4(4.4)$ & $0(0.0)$ & $13(14.4)$ \\
76 a 80 & $4(4.4)$ & $0(0.0)$ & $0(0.0)$ & $4(4.4)$ \\
Más de 80 & $0(0.0)$ & $3(3.3)$ & $0(0.0)$ & $3(3.3)$ \\
Total & $68(75.6)$ & $21(23.3)$ & $1(1.1)$ & $90(100)$ \\
\hline
\end{tabular}


Tabla 2: Diagnóstico.

\begin{tabular}{lccc} 
Diagnóstico & $\begin{array}{c}\text { Masculino } \\
\mathrm{n}(\%)\end{array}$ & $\begin{array}{c}\text { Femenino } \\
\mathrm{n}(\%)\end{array}$ & $\begin{array}{c}\text { Total } \\
\mathrm{n}(\%)\end{array}$ \\
\hline $\begin{array}{l}\text { Trastorno } \\
\text { muscular (I) }\end{array}$ & $9(10.0)$ & $44(48.9)$ & $53(58.9)$ \\
$\begin{array}{l}\text { Trastorno } \\
\text { articular (II) }\end{array}$ & $5(5.6)$ & $31(34.4)$ & $36(40.0)$ \\
$\begin{array}{l}\text { Trastorno } \\
\text { hipomovilidad (III) }\end{array}$ & $0(0.0)$ & $1(1.1)$ & $1(1.1)$ \\
Total & $14(15.6)$ & $76(84.4)$ & $90(100.0)$ \\
\hline
\end{tabular}

tegral, en un local habilitado para la realización del estudio, con las condiciones mínimas indispensables. Se utilizó luz artificial, espejo bucal y explorador, depresores linguales, regla milimetrada, medidor de Willys, estetoscopio y lápiz estilográfico. El examen físico se realizó de manera individual con los pacientes sentados en silla escolar.

En el estudio se consideraron variables sociodemográficas como sexo y edad, y clínicas como trastorno temporomandibular diagnosticado según clasificación de Okeson con los parámetros clínicos dolor, limitación de la apertura bucal, desgaste dentario, ruidos articulares y factores etiológicos presentes como ausencia de rehabilitación protésica, rehabilitación protésica inadecuada, hábitos y macrotraumas. ${ }^{7}$

Considerándose factor etiológico todo aquel hallazgo referido por el paciente en el interrogatorio y/o el constatado en el examen clínico en el sillón dental por el examinador.

Los signos y síntomas de los trastornos temporomandibulares fueron detectados objetivamente durante la exploración clínica, donde se valoraron los músculos, las ATM y los dientes, considerando todos los que presentaba el paciente mediante la realización de un examen clínico estandarizado siguiendo la rutina descrita por Okeson. ${ }^{7}$

Para la recabación de información se utilizó una planilla de recolección de datos individual confeccionada para el efecto que fue llenada en presencia del paciente mediante el interrogatorio y el examen físico.

\section{RESULTADOS}

En la Tabla 1, considerando la clasificación de Okeson para los trastornos temporomandibulares, hubo un predominio del trastorno temporomandibular del grupo I correspondiente al trastorno muscular con $75.6 \%$, siendo el rango de 60 a 65 años el más afectado con $41.2 \%$.

En la Tabla 2 se evidencia que hubo un predominio general del sexo femenino en los pacientes afectados por los trastornos temporomandibulares, siendo el trastorno muscular el más significativo con $48.9 \%$, seguido del articular con 34.4\%.

En la Tabla 3 se evidencia que existió una variedad de factores etiológicos y una coexistencia de ellos en un mismo paciente. El hábito de bruxismo fue detectado en $65.5 \%$ de los pacientes predominando en ambos sexos, en sentido general se puso de manifiesto un predominio de factores etiológicos relacionados con el desdentamiento y sus secuelas en la rehabilitación protésica.

En la Tabla 4 se muestran los parámetros clínicos más frecuentemente referidos en la literatura y de ellos el desgaste dentario fue el más reportado de forma general, presentándose en ambos tipos de trastornos con $83.3 \%$, el dolor aparece en ambos grupos como otro parámetro predominante.

\section{DISCUSIÓN}

La cantidad de pacientes afectados se manifestó con una disminución gradual del total de pacientes según aumentaba la edad. Investigaciones han demostrado

\section{Tabla 3: Etiología.}

\begin{tabular}{lccc} 
Factor etiológico & $\begin{array}{c}\text { Masculino } \\
\mathrm{n}(\%)\end{array}$ & $\begin{array}{c}\text { Femenino } \\
\mathrm{n}(\%)\end{array}$ & $\begin{array}{c}\text { Total } \\
\mathrm{n}(\%)\end{array}$ \\
\hline $\begin{array}{l}\text { Ausencia de } \\
\text { rehabilitación } \\
\text { protésica }\end{array}$ & $10(71.4)$ & $36(47.3)$ & $46(51.1)$ \\
$\begin{array}{l}\text { Rehabilitación } \\
\text { protésica inadecuada }\end{array}$ & $3(21.4)$ & $19(25.0)$ & $22(24.4)$ \\
$\begin{array}{l}\text { Hábito bruxismo } \\
\text { Hábito de masticación } \\
\text { asimétrica }\end{array}$ & $7(50.0)$ & $27(35.5)$ & $34(37.8)$ \\
$\begin{array}{l}\text { Hábito empuje lingual } \\
\text { Hábito de onicofagia }\end{array}$ & $3(21.4)$ & $17(22.3)$ & $20(22.2)$ \\
$\begin{array}{l}\text { Hábitos posiciónales } \\
\text { Macrotraumas }\end{array}$ & $2(14.2)$ & $5(6.5)$ & $6(6.6)$ \\
\hline
\end{tabular}

Nota: El \% de cada sexo se calcula según el total del género para las columnas. 


\begin{tabular}{lcc}
\multicolumn{3}{c}{ Tabla 4: Parámetros clínicos. } \\
\hline $\begin{array}{l}\text { Trastorno } \\
\text { muscular } \\
\mathrm{n}(\%)\end{array}$ & $\begin{array}{c}\text { Trastorno } \\
\text { articular } \\
\mathrm{n}(\%)\end{array}$ \\
\hline Parámetro clínico & $40(75.4)$ & $21(58.3)$ \\
\hline $\begin{array}{l}\text { Dolor } \\
\text { Sonidos articulares }\end{array}$ & $36(67.9)$ & $23(63.8)$ \\
$\begin{array}{l}\text { Restricción del } \\
\text { movimiento } \\
\text { mandibular }\end{array}$ & $31(58.4)$ & $13(36.1)$ \\
$\begin{array}{l}\text { Desgaste dentario } \\
\text { Otros }\end{array}$ & $43(81.1)$ & $20(55.6)$ \\
\hline
\end{tabular}

mayor prevalencia de TTM con el incremento de la edad de los pacientes, sugiriendo mayor tendencia a padecer patologías de la ATM como consecuencia de un deterioro de la salud oral y general o cambios degenerativos osteoarticulares en el organismo. ${ }^{8}$

Consideramos que este pensamiento es una visión marcada por otros momentos del desarrollo social donde las expectativas de vida limitaban con menos edad, pero en los tiempos actuales donde un número importante de países ha elevado estas expectativas sobre los 80 años, y dentro de ellos Cuba, se impone manejar profesionalmente estos enfoques, lo cual podría ser la razón que ponga en evidencia una diferencia de nuestros resultados con otros autores revisados. ${ }^{9}$

La mayoría de los autores concuerdan en que los problemas más frecuentes en los trastornos temporomandibulares son los desórdenes funcionales de los músculos de la masticación y de las articulaciones temporomandibulares. ${ }^{10}$

Muchos autores reconocen un predominio del grupo I y II en la población general, al establecer los diferentes grupos Okeson plantea la prioridad de los padecimientos musculares. $^{7}$

Coincidiendo con estos planteamientos, también otros estudios reportan la presencia femenina en mayor cuantía, diversas explicaciones se han aplicado para justificar esta diferencia de género en la patología destacando, entre otras, la ubicación más posterior del cóndilo mandibular antropológicamente en el sexo femenino. ${ }^{11}$

La existencia de factores estrógenos dependientes en las articulaciones temporomandibulares de las mujeres o la mayor sensibilidad de éstas ante el cotejo signo-sintomatológico que acompañan a los trastornos temporomandibulares es otra explicación. ${ }^{12}$
La etiología de los trastornos temporomandibulares es reconocida, tanto en el ámbito de la literatura internacional como en estudios nacionales, como multifactorial y resulta impreciso definir un orden de prioridades en uno $\mathrm{u}$ otro factor.

Numerosos estudios demuestran la influencia de otros hábitos tales como la onicofagia, el empuje lingual, la succión del pulgar en pacientes con trastornos temporomandibulares en edades más jóvenes, así lo confirman estudios realizados en la Facultad de Estomatología de La Habana y estudios en adolescentes y jóvenes realizados en nuestro país también son reportados en la literatura médica. ${ }^{13}$

Estudios realizados por Sardiña y colaboradores en $2010^{14}$ demuestran que las alteraciones oclusales tales como la pérdida de dientes y la masticación unilateral que ésta puede generar constituyen factores de alto riesgo en la población adulta general que ellos estudiaron.

Según Yun y su equipo en $2005^{15}$ los adultos mayores sufren a menudo traumas físicos y asumen que resulta innegable que un traumatismo directo importante en la mandíbula, como un golpe en el mentón, puede producir instantáneamente un trastorno intracapsular. Si este traumatismo se produce con los dientes separados (traumatismo de boca abierta), el cóndilo puede experimentar un desplazamiento brusco en la fosa articular.

En un trabajo realizado por Kim y colaboradores en $2010^{16}$ se plantea que los macrotraumatismos pueden producirse también cuando los dientes están juntos, es decir, traumatismo de boca cerrada. Si la mandíbula recibe un golpe cuando los dientes están juntos, la intercuspidación dental mantiene la posición mandibular, evitando el desplazamiento articular. El traumatismo con la boca cerrada es pues, menos nocivo para el complejo cóndilo-disco.

Los trastornos temporomandibulares se caracterizan clínicamente por dolor, limitación en la apertura bucal, sonidos articulares y desgaste dentario, pero sin duda el síntoma más frecuente por el cual las personas buscan tratamiento es el dolor, lo cual coincide con los resultados de este estudio en pacientes de la tercera edad donde los examinados refirieron este síntoma.

En investigaciones desarrolladas en la Facultad de Estomatología de La Habana los sonidos articulares fueron el signo más común; sin embargo, las cifras de esta investigación muestran que la presencia de sonidos articulares se observó en un tercer nivel inferior de manifestación, aunque constituyen un signo clínico patognomónico, coincidiendo con las reportadas por Grau. ${ }^{13}$

La limitación de los movimientos mandibulares se manifiesta en un porcentaje considerable de los afectados con trastornos temporomandibulares. 
Es necesario enfatizar que en el adulto mayor también están coincidiendo procesos inflamatorios y degenerativos de forma sistémica que pudieran provocar estas limitaciones con causa orgánica.

\section{CONCLUSIONES}

Se concluye que el rango de edad entre 60 y 65 años y en sexo femenino fue donde más prevalecieron los trastornos temporomandibulares y el trastorno más frecuente presentado fue el muscular grupo I, según la clasificación de Okeson. De los factores etiológicos oclusales encontrados, el bruxismo y la rehabilitación protésica son los más representativos. El desgaste dentario fue el parámetro clínico más reportado de forma general presentándose en ambos tipos de trastornos.

\section{BIBLIOGRAFÍA}

1. Ollar J. Envejecimiento y función cognitiva. De la declinación al deterioro. En: Mangone CA, Allegri R. Demencia: enfoque multidisciplinario. Buenos Aires: Sagitario Editores; 1997. pp. 71-97.

2. Promoción y prevención en salud a medida que las personas envejecen. Geroinfo [Revista en Internet]. 2013 [Citado 18 Feb 2015]; 8 (3): [aprox. 2 p]. Disponible en: http://www.medigraphic. com/pdfs/geroinfo/ger-2013/ger133a.pdf.

3. Taboada O, Gómez Y, Taboada S. Prevalencia de signos y síntomas de los trastornos temporomandibulares en un grupo de adultos mayores. ADM [Revista en Internet]. 2004 [Citado 12 Mar 2015]; 61 (4): [Aprox. 12p]. Disponible en: http://www.medigraphic.com/ pdfs/adm/od-2004/od044b.pdf.

4. Witulski S, VogI TJ, Rehart S, Ottl P. Evaluation of the TMJ by means of clinical TMD examination and MRI diagnostics in patients with rheumatoid arthritis. Biomed Res Int. 2014; 2014: 328560.

5. Granizo LR. Cirugía oral y maxilofacial. Editora Barcelona, Madrid, 1997.

6. Cabo R, Grau I, Sosa M. Frecuencia de trastornos temporomandibulares en el área del Policlínico Rampa, Plaza de la Revolución. Facultad de Estomatología «Raúl González Sánchez». Rev Haban
Cienc Méd. 2009; 8 (4). Disponible en: http://scielo.sld.cu/scielo. php?pid=S1729-519X2009000400011\&script=sci_arttext.

7. Okeson JP. Tratamiento de oclusión y afecciones temporomandibulares. 7a ed. Barcelona Elsevier, 2013, pp. 233-242.

8. Rojas MM, Silveira P, Martínez LM. Gerontología y Geriatría, un recuento de poco más de un siglo. Acta Médica del Centro [Revista en Internet]. 2014 [Citado 12 Mar 2015]; 8 (1): [aprox. 6 p]. Disponible en: http://www.revactamedicacentro.sld.cu/index. php/amc/article/view/49/html.

9. Thilander B, Rubio G, Pena L, de Mayorga C. Prevalence of temporomandibular dysfunction and its association with maloclussion in children and adolescents: an epidemiologic study related to specified stages of dental development. Angle Orthod. 2010; 72 (2): 14.

10. Araneda P, Oyarzo JF, González M, Figueroa C. Intervención psicológica en trastornos temporomandibulares: revisión narrativa. J Oral Res. 2013; 2 (2): 86-90.

11. Sena M, Mesquita K, Santos F, Silva F, Serrano K. Prevalence of temporomandibular dysfuntion in children and adolescents. Rev Paul Pediatr. 2013; 31: 538-545.

12. Boscato RC, Almeida CD, Koller AA, Presta ML. Goettems. Influence of anxiety on temporomandibular disorders-an epidemiological survey with elders and adults in Southern Brazil. J Oral Rehabil. 2013; 40: 643.

13. Salomón BO. Oclusión y trastornos temporomandibulares en estudiantes de estomatología (Trabajo para optar por el título de Especialista de Primer Grado en Prótesis Estomatológica) 2003 FCM "Mariana Grajales Coello" Holguín.

14. Sardiñas M, Casas J. Anomalías de la oclusión dentaria asociadas a la disfunción temporomandibular. [En línea]. Rev Med Electrón. 2010; 32 (3). [Consultado marzo 2012] Disponible en: http://www.revmatanzas. sld.cu/revista\%20medica/ano\%202010/vol3\%202010/ema6.htm.

15. Yun PY, Kim YK. The role of facial trauma as a possible etiologic factor in temporomandibular joint disorder. J Oral Maxillofac Surg. 2005; 63 (11): 1576-1583.

16. Kim HI, Lee JY, Kim YK, Kho HS. Clinical and psychological characteristics of TMD patients with trauma history. Oral Dis. 2010; 16 (2): 188-192.

Correspondencia:

Licet Abreu-García

E-mail: licet.abreu@nauta.cu 